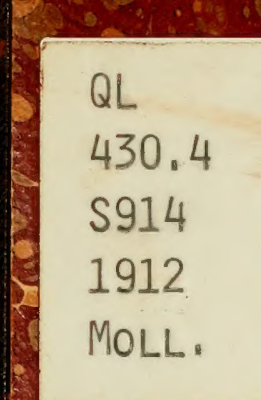




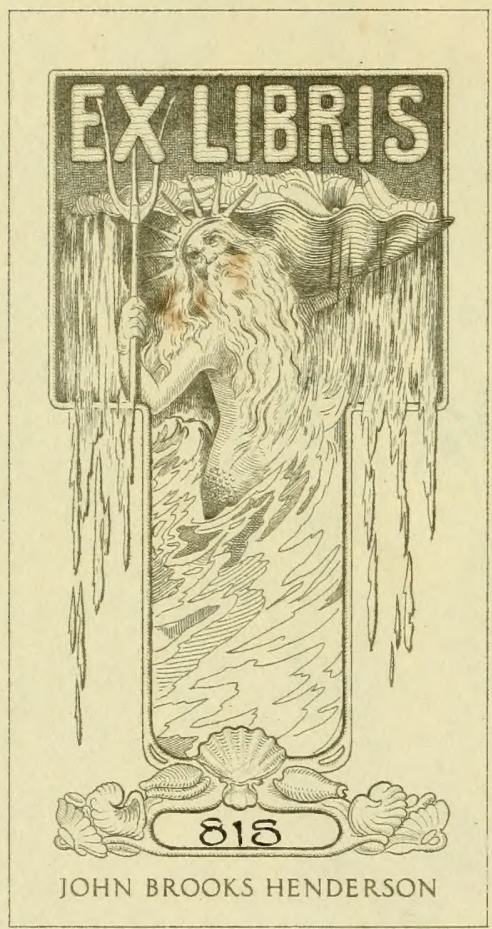

I stres. 


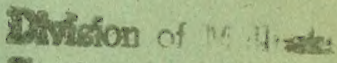
Sectional Gibrary

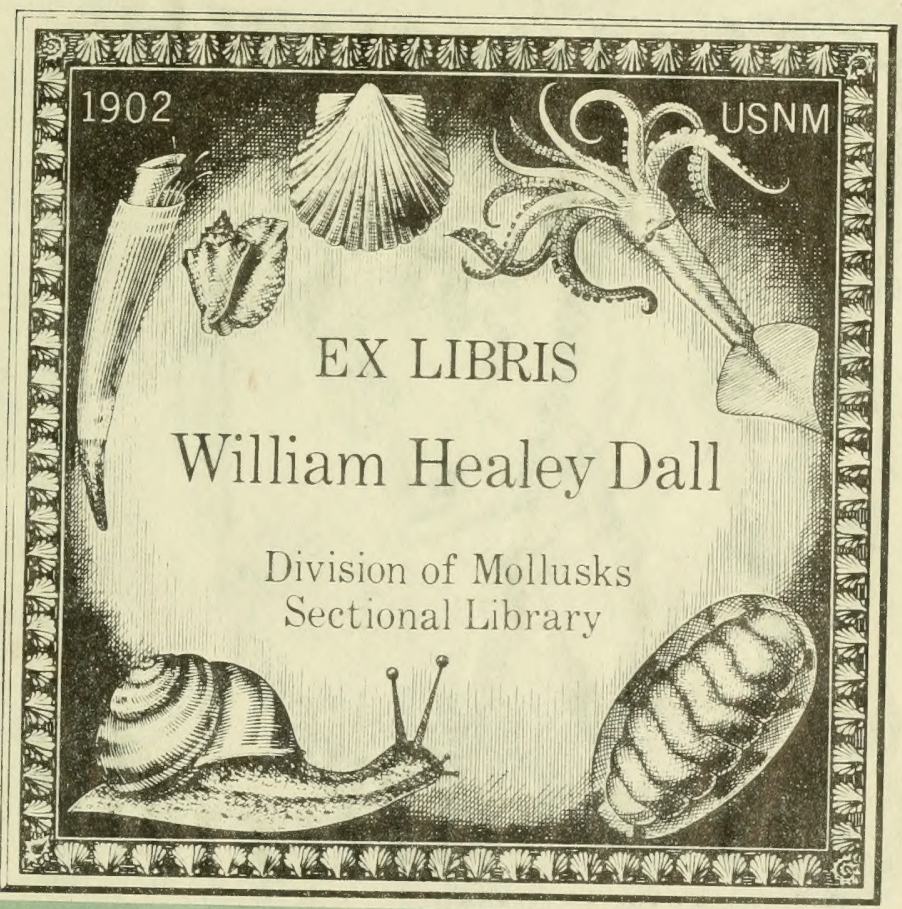







\title{
Bemerkungen zu den
}

\section{Clavatula-Gruppen Perrona und}

\section{Tomella.}

\author{
Von \\ Hermann strebel.
}

Dividion of Molluedes

Fetectional Library

II it einer Tafel.

Aus „Mitteilungen aus dem Naturhistorischen Museum“. XXIX.

(2. Beiheft zum Jahrbuch der Hamburgischen Wissenschaftlichen Anstalten. XXIX.)

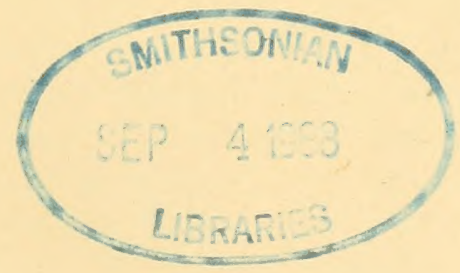

Hamburg 1912.

Kommissionsverlag von Lucas Gräfe \& Sillem 

Den Anlaß zu der vorliegenden Studie gibt das von Kapitän HuPFER von der WOERMANN-Linie auf seinen Fahrten an der Westküste Afrikas mit vieler Umsicht in den Jahren 1887-1891 für das Naturhistorische Museum in Hamburg gesammelte, reiche und schöne Material, das noch einer eingehenden Bearbeitung harrt. Gelegentlich der Umordnung unserer Sammlungen fiel mir bei den hier zu behandelnden beiden ClavatulaGruppen Perrona und Tomella das aus jenen Reisen stammende reichhaltige Material auf, das zu einer besonderen Bearbeitung aufforderte, da es nicht nur für die bekannten Arten manches Neue bot, sondern auch einige neue Formen enthielt.

Einleitend einige allgemeine Bemerkungen. Soweit mir bekannt ist, sind nach WEINKAUFF in MARTINI \& CHEMNITZ, II. Edit., und TRYON in Manual of Conchology keine neueren Spezialbearbeitungen dieser ClavatulaGruppen erschienen. Für ihre systematische Stellung kommen außerdem noch P. Fischer, Manuel de Conchyliologie und neuerdings M. Cossmans, Essaies de Paléoconchologie comparée, Heft II, 1896, in Betracht. WEINKAUFF gibt keine systematische Zusammenstellung der Pleurotomen, bezeichnet aber die hier zu behandelnden Arten alle als Clavatula. TrYoN faßt unter Clavatulinae die Gattungen Clavatula (mit den Untergattungen Perrona und Clionella), Pusionella und Surcula zusammen. FIsCHER gibt, meiner Ansicht nach mit Recht, sowohl Pusionella wie Surcula eine selbstständigere Stellung, ebenso Cossmaxs. Abgesehen von der fraglichen Stellung der Pusionella, die Cossmann zu den Terebridae zählt, wenn auch als Übergang zu Clavatula, muß, wie schon Cossmanx hervorhebt, Surcula seiner abweichenden Embryonalwindungen halber abgesondert werden, während, wie ich hinzufügen möchte, in dieser Beziehung Clavatula, Perrona und Tomella unter sich gleichartig sind.

Faßst man nur das wichtige Merkmal der Embryonal- und Postembryonalwindungen ins Auge, für deren Feststellung es freilich guterhaltenen Materials bedarf, das verhältnismäßig selten geboten ist, so würden sich wahrscheinlich nicht alle die in TRYON angeführten Arten als in die dafür angenommene Gattung gehörig erweisen. So finde ich 
z. B. in der Gattung Surcula, daß die darin aufgeführte S. fulminata KIEN. in die Clavatula-Gruppierung gehört, wie denn auch den übrigen Charakteren nach, manche der Arten eine andere Gruppierung notwendig machen diirften. Das gleiche gilt aber in dieser letzteren Beziehung von den in Clavatula, Perrona, Tomella, Clionella aufgeführten Arten, worauf ich noch zurückkomme, soweit es sich um die allein hier berücksichtigten Gruppen Perrona und Tomella handelt. Wenn nun auch die oben erwähnte Gleichartigkeit im Typus der oberen Windungen ein Zusammenfassen der Gruppen Clavatula, Perrona und Tomella unter einem Kollektivnamen zu empfehlen scheint, so habe ich hier doch nicht zu entscheiden, ob man dazu TRYONs Bezeichnung "Clavatulinae“ wählen soll. Dem fossilen Torkommen nach scheinen Clavatula und Perrona gleichalterig (mittleres Miozän) zu sein. Ebensowenig will ich entscheiden, ob diese Gruppen als Gattungen oder Untergattungen gelten sollen, denn zu alledem bedarf es auch noch der Feststellung des anatomischen Befundes (nicht nur der Radula), der ja erst für die wenigsten Arten festgestellt ist. Ich wähle deshalb die indifferentere Bezeichnung Gruppe.

Im Gegensatz zu TRYON teile ich FIschers Ansicht, daß Perrona und Tomella trotz ihrer in mancher Beziehung gleichartigen Charaktere, wenn auch nur als Gruppen, voneinander getrennt zu halten sind. Auch Cossmann, der 1. c. p. 68 in der Überschrift Tomella als gleich Perrona anführt, bemerkt am Fuße, daß Tomella lineata eine Sonderstellung einnimmt, und, was wichtig ist, daß es dafür keine fossile Formen gibt. Ich möchte hierzu noch folgendes bemerken. BeiDurchsicht der in Cossuanvl.c. und in BELLARDI-SACCO (Molluschi del Piemonte e della Liguria) gegebenen Abbildungen fossiler Formen finde ich, daß die unter Perrona verzeichneten Arten: jouanneti, bicarinata und semimarginata sich weniger gut dem Typus Perrona spirata LAM. ampassen lassen, als z. B. laciniata, gotica und carinifera, die als Clavatula aufgeführt werden. Alle diese im oberen und mittleren Miozän vorkommenden Arten geben jedenfalls Anhaltspunkte für das Alter und die ursprüngliche Verbreitung der jetzt noch lebenden analogen Formen, während es nach den bisherigen fossilen Funden scheint, als ob dem Typus zu Tomella und wohl auch den sich ihm anschließenden Formen nur eine rezente Entstehung zukommt.

Ich will hier noch einschalten, daß in C. SEMPER, Reisen im Archipel der Philippinen, vol. 9, sich eine Arbeit von R. BERGH befindet: Malacologische Untersuchungen, Teil VI, Lief. 3, Tectibranchiata-Pectinibranchiata 1908, worin die Anatomie von P. lineata und spirata enthalten sein soll. Zu der Anordnung des mir vorliegenden Materials bemerke ich, daß zur besseren Übersicht der geographischen Verbreitung der Arten die Fundorte durchgehend von Norden nach Osten bezw. Süden fortschreitend angeordnet sind. Die Fundorte sind bald auf der Ausreise, bald auf der 
Rïckreise von Kapt. HuPFER besucht. Einzelne derselben waren auf den Karten nicht verzeichnet, doch ergal) sich deren Lage meist mit Sicherheit aus der Reihenfolge der Sammeldaten. Über die richtige schreibweise einzelner Fundorte scheinen die Ansichten auseinander zu gehen. Die genauen Daten, an welchen die Funde gemacht sind, waren nicht immer festzustellen, oft mußte an deren Stelle Jahr und Ilonat im Eingangskatalog ausreichen. Aber für meine Zwecke kommen die Daten weniger in Betracht, als die Gleichheit des Fundortes.

Da das Hauptmaterial, welches für die vorliegende Arbeit benutzt wurde, von Kapt. HuPFER stammt, so habe ich nur bei den wenigen Stuicken, die eine andere Herkunft haben, eine entsprechende Angabe gemacht.

Bei Anführung der Maße wolle man in der ersten Zahl die Anzahl der Windungen, in der zweiten die ganze Höhe, in der dritten die Höhe der letzten Windung, in der vierten die Breite des Gehäuses an der letzten Windung finden.

\section{Gruppe Tomella SWATNSon.}

WILLIAj StVAinson. A 'Treatise on Malacology or Shells and Shellfish 1840, pag. 314. ,Shell fusiform, smooth; the spire of very few whorls (?) and not longer than the channel; inner lip with a thick callosity at the top; the slit short and wide." Species: lineata, Encycl. meth., tab. 440, fig. 2 ; clavicularis, ibid. fig. 4 ; filosa, ibid. fig. 6 ; lineolata, ibid. fig. 11 . Die letzteren 3 LAJARCKschen Arten sind fossil und gehören entschieden nicht zil Tomella.

PaUl Fischer 1. c. Subgen. Tomella. Spire lisse, sinus large, placé presque au milieu du bord labial (C. lineata Lam.).

G. W. TRyon, Manual, vol. VI, p. 231, will Tomella nicht von Perrona getrennt wissen, weil einerseits die Spira nicht immer glatt ist (im Gegensatz zu gekielt bei Perrona), und weil die Lage des Sinus nur durch den Callus oben an der Mündung so weit nach unten gerückt ist.

Cossmany 1. c. Das über die Gruppe Gesagte ist schon weiter vorne angeführt.

Die oben wiedergegebene Diagnose von SWAINSOx ist schon durch die Bemerkung „sehr wenige Windungen“ irreführend und an sich nichtssagend. Auch das Hinzufïgen der fossilen Formen verwirrt das Bild, das man sich nach dem Typus lineata von der Gattung machen soll. FIsCHERs kurze Diagnose bezeichnet nur den Gegensatz zu Clavatula, dem an sich trifft die Bezeichnung "spire lisse" nicht zu. "IRYoNs Begriindung, weshalb er Perrona und Tomella zusammenwirft, ist nur auf lineata begriindet und ist in dem Falle durchaus unzutreffend. Andererseits bringt 'T'RYON 
anch obesa REEve und taxus CHEMN. in die Gattung Perrona, wodurch eine Tielseitigkeit der Schalencharaktere entsteht, die jede Kemneichnung natiirlicher Gruppen ummöglich macht. Damn kömnte man überhaupt Perrona und Tomella ausschließen und alle dahin gerechneten Arten zu Clavatula zählen, eine Gattung, die schon nach TrYoNs Zusammenstellung sehr Verschiedenartiges enthält.

Da nun die vorhandenen Diagnosen ungenïgend sind, so gebe ich nachstehend eine neue, zumal einige neue Formen hinzukommen.

Gruppe Tomella SwaINs. emend.

Gehäuse spindel- bis keulenförmig, festschalig, ziemlich glänzend, das Gewinde ist mehr weniger niedriger als die letzte Windung. Die 10 bis 11 Windungen nehmen anfangs langsamer, später mehr weniger rascher an Breite zn, so daß der Seitenkontur des Gewindes mehr weniger eingebogen erscheint. Sie sind anfangs nach unten etwas vorgewölbt, später gleichmäßiger und wenig vorgewölbt. Je nach der allgemeinen Form setzt sich die letzte Windung stark oder wenig vorspringend rom Gewinde ab, die unteren Windungen gehen zuweilen nur etwas wulstig oder auch steil dachförmig von der Naht ab, so daß sich im letzteren Falle beim Übergang in die Wölbung eine Kante bildet, die aber wenig merkbar vorspringt. Die Windungen sind durch eine einfache Naht getrennt, die auf den oberen Windungen immer durch einen Wulst berandet ist, der später aufhört (lineata) oder aber sich in einen gekielten Wulst umändert, entsprechend den Formen, deren untere Windungen sich dachförmig von der Naht absetzen. Auf den Nahtwulst pflegt, am deutlichsten auf der letzten Windung, eine seichte Einschnïrung zu folgen, auf diese dann die Zone, in der sich an der Mündung der sogenamnte Analeinschnitt befindet, der nach hinten abgerundet, nach vorne verbreitert ist. Der Schnabel setzt sich immer deutlich von der Wölbung der letzten Windung ab und ist unten gedreht vorgewölbt, so daß hier die Anwuchsstreifen quer verlaufen. Die Mündung ist oval, nach oben zugespitzt, nach unten in den offenen Kanal anslaufend, der Spindelrand ist entsprechend oben eingebogen, mit einem schmalen, dïnnen, meist nur nach unten deutlichen Callusbelag versehen. Oben an der Mündung tritt ein im Innern unterhalb der Naht verlaufender Callusstreifen als mehr weniger ausgedehnter Wulst hervor. Das Innere der Mündung ist nur mit einem dünnen, weißlichen Belag versehen. der die äubere Färlung durchschemen läbt. Der Mundrand ist scharf. Die ersten $1^{1 / 2}$ Windungen sind glänzend, glatt und durchscheinend, damn tritt die Skulptur auf, die einerseits ans Spiralfurchen besteht, die sich bei lineata bis zuletzt ziemlich scharf ausgeprägt erhalten, bei anderen 
Formen sich abschwächen und nur bei starker Tergrößerme sichtbar sind, auch zuweilen weitläufiger gereiht stehen. Anderseits treten Falten auf, die, der Anwuchsrichtmig folgend, geschweift sind, anfangs sich über die ganze Windung erstrecken, damn oben obsolet werden und schließlich in kurze schräge Falten oberhalb der Naht auslaufen, die aber je nach der Art nur zwischen der zweiten und siebenten Windung anftreten, dam ganz verschwinden. An Schmabel befindet sich immer eine Reihe mehr weniger scharf ansceprägter und mehr weniger weitläufig gereihter Spiralreifchen.

Der Deckel ist oval, an der nach innen gekehrten Seite stumpfwinkelig, weil hier in der Mittelhöhe der Kernpunkt liegt. Nicht nur in FISCHER 1. c. p. 590 ist der Deckel verkehrt herum abgebildet, d. h. mit dem Kernpunkt an der rechten Seite, sondern ich fand ihn auch mehrfach in gekauften Stiicken verkehrt eingeklebt.

\section{Tomella lineata LAJ.}

Figg. 1, 1a, b, 2, 3, 5.

LAJIARCK 182.2, Anim. s. vert., vol. VII, p. 93, Pleur. lineata mit var. castanea, fusco lineatu, mit Hinweis auf Encyclop. meth., pl. 440, Figg. 2a, b.

Ders., Edit. Deshayes 1840 , vol. IX, p. 348.

Jiartini \& Chemn., Conchyl. Cab. Supplement vol. XII von Schubert und WAGNer 1827, p. 156, Taf. 234, Figg.4104a, b. Nur die dunkle Varietät. Edit. II, WeInkaufF, 1. 120, Taf. 26, Figg. 5, $8-11,14$.

KieNeR, Icon., vol. 4, p. 47, Taf. 22, Fig. 1.

ReEve, lcon., Taf. 11, Figg. 96a-c.

Tryox, JIanual, vol. VI, p. 231, Taf. S, Figg. 10, 11.

Die wesentlichen Merkmale dieser Art sind schon in der Beschreibung: der Gruppe angegeben. Hier nur die besonderen Merkmale. Die große Terschiedenheit der Form ist in meinen und WEINKAUFFs Abbildungen zur Genïge wiedergegeben. Die Färbung ist hell melonengelb, nach oben zu lebhafter gefärbt, mit schmalen braunen Linien in der Auwuchsrichtung verziert, die nicht sehr regelmäßig gereiht sind, anch zuweilen sich verzweigen. Der wulstige weiße Callus an der Müindung überragt an größeren Stücken meist noch die Naht. Auf der letzten Windung ist zuweilen die Naht schmal weißlich berandet, auch der Spindelbelag ist unten weiß. Die braune Varietat tritt, wie es scheint, viel seltener anf;

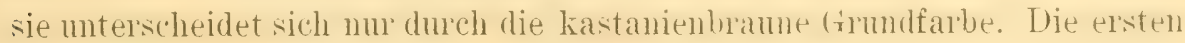
$1 \frac{1}{2}$ Windungen sind zuweilen violett abschattiert, die schon geschilderte Faltenskulptur erstreckt sich über die folgenden 3 Windungen (Fig. 1a). Die Spiralfurchen werden zuweilen bei großen Stïcken auf der Mittelpartie der letzten Windung seln undeutlich. 
Die bei weitem vorwiegende Form ist die keulenförmige (Fig. 1), deren Gewinde einen ziemlich stark eingebogenen Seitenkontur zeigt und deren letzte Windung sich wulstig ron der Naht absetzt, damn mehr oder weniger deutlich oberhalb der Analzone eine Einschnürung zeigt. Um Wiederholungen bei den Einzelaufführungen zu vermeiden, bezeichme ich diese Form mit $A$.

Selten auftretend ist eine Form (Fig. 2), die weniger keulenförmig erscheint, weil der Seitenkontur des Gewindes wenig eingebogen ist, und weil schon die letzten Windungen sich wulstig von der Naht absetzen. Diese Abweichung bezeichne ich mit $B$, und mit $B 1$ die ebenfalls selten auftretende, sehr ähnliche, aber schlankere Form (Fig. 5), die sich der folgenden Form nähert.

Ich bemerke noch, daß bei jungen Stücken sich ihre Zugehörigkeit zu einer oder der anderen der vorstehend verzeichneten Formen nicht bestimmen läßt.

\section{T. lineata var. macilis.}

Figg. 4, 4 a.

Diese Form ist spindelförmig und der Seitenkontur des Gewindes ist kaum eingebogen. Die unteren Windungen setzen sich dachförmig von der Naht ab, so daß sie beim Übergang in die Windungswölbung. eine Kante bilden (Fig. 4a). Alle uibrigen Charaktere sind die gleichen wie die der vorangehend geschilderten Formen.

Ich sondere diese Form als Varietät ab, weil sie, wie aus dem nachfolgenden allgemeinen Verzeichnis ersichtlich ist, wo sie mit $C$ bezeichnet wird, mit nur "2 Ausnahmen in den nördlichsten Fundorten vorkommt, wo die Hauptform und ihre seltenen Abweichungen nicht rorzukommen scheinen.

Verzeichnis der Fundorte und des daselbst gefundenen

Materials von $T$. lineata und ihrer Tarietät.

1. Gorée. Nr. 25345 . 30.6. 1888. Salzwasser, 12 Faden. 2 Stiicke $C$. Figg. 4, 4 a. $10-23,8-14,3-7,9$.

2. Waterboo. Nr. 14498. Aug. 12. 1887. 9 Faden, Sand und Mudd-Grund. 1 Stïck $C$.

3. Grand Bassa.

Nr. 14509. Aug. 12. 1887. 8 Faden. 1 Stiick $C$.

Nr. $25266 . \quad$ Septbr. 15. 1888. 8 Faden, Sand und Mudd-Grund. 1 Stiick $C$, mit Pagurus besetzt.

4. Little Pootan. Nr. 14466. Aug. 11. 1887. 14 Faden, MuddGrund. :3 Stiicke $C$. 
5. Accra. Nr. 25210. 15. 9. 1888. Salzwasser, 4¹/2 Faden. 1 Stiick $B$ 1, 4 Stiicke $C$, alle mit Pagurus besetzt.

6. Salt Point (Saltpond). Nr. 25 189. Septbr. 1. 1888. Salzwasser, 4\% Faden. 1 Stïck $B$, mit Pagurus besetzt.

7. Grand Popo.

Nr. 25170. Septbr. 1888. Salzwasser, 6 Faden. 1 Stïck $C$.

Nr. 25173. Septbr. 1888. Salzwasser, 6 Faden. 1 Stiick $C$.

Nr. 25484. Septbr. 1888. Salzwasser, 6 Faden. 5 Stïcke $C$.

8. Bugama (Boguma). Nr. 25404. Septbr. 1888. Im Fluß, beinahe frisches Wasser. 1 Stiick jung. Leider fehlt bei dieser Nummer die 'Tiefenangabe, doch erinnert PROF. DR. PFEFFER genau, daß, da unter dem Material sich auch Tiere befanden, die nur in Salzwasser leben

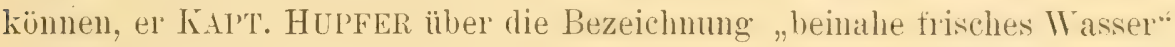
interpelliert hat, der ihm sagte, man habe dort von der Oberfläche trinkbares Wasser geschöpft. Es wird also wohl in der 'Tiefe sich ein Strom salzigen Wassers hineingezogen haben.

9. Kamerun. $\mathrm{Nr}^{2}, 24533$. Juni 1888. Frisches Wasser, grober Sandboden. S Stücke, darunter viele junge, die größeren gehören zur Form A. Auch hier fehlt die 'liefenangabe, aber mit Bezug auf die Angabe "frisches WVasser" gilt das vorstehend Gesagte.

10. Black Point (Gaboon).

Nr. 26874. 16. 4. 1889. Salzwasser, 5 Faden. 5 Stïcke $A$, mit Pagurus besetzt, darunter 1 abnorm gebildetes.

Nr. 26876. Desgleichen. 1 Stïck $A$.

11. Setta Cama (Setta Kama).

O. Nr. 4 Stiicke $A$.

O. Nr. Mai 1891. 8 Faden, Schlammgrund. I Stiick $C$.

12. Fluß Nyango.

Nr. 24 329. 16.3. 1888. Salzwasser, 7 Faden, Mudd-Grund. 1 Stïck brame Valietait $B 1$.

$$
10^{1 / 2}-30,1-20,6-10,1 \text {. }
$$

Nr. 26789. 1888. Salzwasser, 6 Faden, weicher Grund. 2 Stiicke $A$.

Nr. 26918. April 1889. Salzwasser, 6 Faden, Mudd-Grund. 4 Stïcke B 1, braune Tarietät.

13. Loango.

$$
\text { Fig. 5. } 10^{1 / 2}-28,0-19,0-8,6 .
$$

Nr. 21255. Mai 1891. Salzwasser, Schlammgrund. 1 Stiick $A$, mit Pagurus besetzt.

Nr. 20550. April 1889. Salzwasser, 41/2 Faden, 1 Strick $A$.

Nr. 24401. Juni 1888. Salzwasser, 5 Faden, Sand- und MischelGrund. $4^{1 / 2}$ Stücke $A$.

14. Landana (Landane). 
Nr. 26941. 1888. 4 Faden, sehr weicher Schlamm. 2 Stücke $B$, davon eins dunkle Tar.

Nr. 26936. April 1889. 4 Faden, sehr weicher Schlamm. 1 Stïck $C$ mit Pagurus besetzt.

Nr. 26555. Febr. 1889. Salzwasser, 4 Faden. 4 Stiicke $A$, mit Pagurus besetzt.

Nr. 26796. 1888. Salzwasser, 4 Faden. I Stïck $A$.

Nr. 26549. 15. 2.1889. Salzwasser, 4 Faden. 6 Stïcke $A$ und $B$, mit Pagurus besetzt.

15. Kabinda (Cabinda).

Nr. 26988. April 1889. Salzwasser, 4 Faden. 3 Stiicke A, darunter

Fig. 3. $10-29,2-20,0-11,6$.

Nr. 26998. Desgleichen. 5 junge Stiicke.

Nr. 26965. 1889. Salzwasser, $3^{1} / 4$ Faden, Mudd-Grund. 5 Stücke, darimter $2 A, 1 B 1$ und 2 junge.

Nr. 24451. Juni 4. 1888. Salzwasser, 31/4 Faden, Mudd-Grund. 1 Stïck $A$.

Nr. 26802. Aug. 1889. Salzwasser, $3^{3} / 4$ Faden. 1 Stiick $A$.

Nr. 26553. 1889. Salzwasser, $3^{3} / 4$ Faden. 1 Stück $A$, mit Pagurus besetzt.

Nr. 26600. 1889. Salzwasser, $3^{3 / 4}$ Faden. 1 Stück $A$, mit Pagurus besetzt.

O. Nr. Salzwasser, $3^{3 / 4}-4$ Faden, Mudd-Grund. Ca. 125 Stücke, mit Aspidosiphon venabulum besetzt, darunter nur ein paar Stücke der Form $B$.

16. Mucula (Muculla). Nr. 25676. 6. 3. 1890. Salzwasser, 4 Faden, Schlammgrund. 4 Stïcke $A$.

17. Kinsembo (Quissembo). Nr. 24468/9. Juni 4. 1888. 7 Faden, Indd-Grund. 4 Stïcke mit Pagurus besetzt, darunter 2 Form $A, 2$ Form $B$.

Fig. 2. Erhalten $10-29,9-20,5-11,2$.

18. Ambris (Ambriz).

Nr. 21257. Mai 1891. 7 Faden, Schlammgrund. 1 Stïck $A$.

Nr. 25774. ? Salzwasser, 7 Faden. 1 Stïck A, mit teilweise breiteren und sehr dunklen Linien.

O. Nr. Novbr. 1890. Salzwasser. 5 Stïcke A, darunter 2 der dunklen Varietait.

Figg. 1, 1 a, b. ca. $11-33,2-24,6-14,8$. 


\section{Tomella hupferi mihi.}

Figg. 6, 6 a, b.

Das glänzende Gehäuse entspricht in der Form und in dem Aufbau der Windungen der $T$. lineata var. gracilis, nur tritt die Umwandlung des Nahtwulstes in die, in eine Kante auslaufende Abdachmo schon von der 4. Windung an deutlich erkennbar auf. Die Faltum der oberen Windungen entspricht derjenigen von $T$. lineata, nur setzt sie sich, nach oben obsolet werdend, bis zur 6. oder 7 . Windung fort, auch fehlt die Spiralskulptur, die erst auf den unteren Windungen muter der Luye als etwas weitläufig gereihte eingeritzte Linien mehr oder weniger deutlich erkennbar ist. Der Schnabel zeigt unten weitlätige grobe Furchen. Miundung, spindelvand. Basalteil des Schnabels verhalten sich der Gruppe entsprechend. Der oben an der Mïndung austretende weiße Calluswulst ist nicht so kräftig wie bei T. lineata Form A und $B$, sondern wie bei del var. gracilis.

Die Färbung ist hell-bräunlich-gelb, von der sich die breiten dunkleren Flammenstreifen, die in der Anwuchsrichtung verlaufen und teilweise gespalten sind, wirkungsvoll abheben. Der Nahtwulst ist mehr weißlich, die Flammenstreiten ziehen sich uber ihn hinweg. anberdem ist aber in der Mittelhöhe der letzten Windung eine gegliederte weibliche Binde erkemnbar.

1. Gorée. Nr. 25348. 30.6.1888. Salzwasser, 12 Faden. 3 jüngere Stiicke.

2. Klein Popo. O. Nr. Novbr. 1890. Salzwasser, 7 Faden. 1 Stïck, ziemlich hell gefürbt.

3. Groß Popo.

Nr. 14591. Dezbr. 1887. 8 Faden, feiner Schlick-Grund. 1 Stïck.

Nr. 25499. Septbr. 1888. Salzwasser, 6 Faden. 3 Stiucke.

Nr. 25 170. Septbr. 1888. Salzwasser, 6 Faden. 1 Stiick, tot gesammelt, mit Bryozoen besetzt.

$$
10-28,0-17,5-8,5
$$

2 Stiicke, mit Pagurus besetzt.

4. Whydah (Dahomey). Nr. 14649. Dezbr. 1887. 61/2 Faden, blater Lehmboden. 1 Stück.

O. Nr. Novbr. 1890. 5 $\frac{1}{1} / 2$ Faden. 6 Stücke, darunter:

Figg. 6, 6 a, b. $\quad 11-27,6-18,0-7,7$.

Nr. 25425. Septbr. 1888. Salzwasser, 51/2 Faden. 6 Stücke, zulmeist jüngere.

5. Victoria (Kamerun). 1888. Salzwasser, 6 Faden, Mndd-Grund. 1 junges Stiick. 


\section{T. hupferi var. fusce milhi.}

Figg. 7, ๆ

Diese unterscheidet sich von der Hauptform nur durch die dunkelkastanienbraune Färbung, auf der nur die Auslänfer der Flammenstreifen auf dem etwas heller gefärbten gekielten Wulst zu erkennen sind, digegen hebt sich die weißliche artikulierte Binde sehr deutlich ab. Der Mundrand ist außen etwas heller abschattiert. Der Callus oben an der Mündung ist bräunlich-weiß.

1. Lome. Nr. 25304. 1888. Salzwasser, 7 Faden. 1 Stück.

Figg. 7, 7a. $11-26,8-15,7-7,8$.

2. Whydah. Nr. 25269. 15. 9. 1888. Salzwasser, 5¹/2 F'aden. 1 Stiick, etwas jünger.

\section{Tomella pfefferi mihi.}

Figg. $8, s a, b$.

Gehäuse spindelförmig, ziemlich glänzend, im Aufbau ganz ähnlich der vorangehenden Art. Die 11 Windungen nehmen gleichmäßig an Breite zu, die oberen sind durch die Falten unten etwas vorstehend, was sich aber mit Abschwächung der Falten allmählich verliert. Der Schnabel setzt sich auch hier deutlich von der Wölbung der letzten Windung ab. Der Nalitwulst entwickelt sich rasch zu der in eine Kante anslaufenden. ziemlich steilen Abdachung. Die auf die $1^{1} / 2$ glatten Embryonalwindungen folgenden Windungen zeigen bei starker Vergrößerung eine deutliche Spiralstreifung (Reifchen?), die sich besonders unterhalb des Nahtwulstes noch bis zuletzt erhält; auf der letzten Windung zeigt die Hauptwölbung nur Spuren weitläufig gereihter, sehr schwacher, feiner Furchen, während der Schmabel wieder mit ausgeprägten feinen Reifen in abnehmender Stärke besetzt ist. Außerdem treten von Anfang an auf der unteren Hälfte der Windungen angeschwollene Falten auf, die erst fast als Knoten erscheinen, dann sich allmählich zu kürzeren schrägen Falten oberhalb der Naht ausbilden, und die sogar etwas die Abdachung des Nahtwulstes heeinflussen. Diese Faltenbildung verschwindet auf der letzten Windung, so daß sie nur bei jüngeren Stücken noch an allen Windungen sichtbar bleibt (Fig. 8b). Der oben an der Mündung austretende Calluswulst entspricht der Breite des Nahtwulstes. Mündungsform. Spindel mit diimem Belag, Analausschnitt und Imudrand entsprechen der Gruppendiagnose, ebenso der Deckel.

Die Färbung ist eine helle bräunliche Fleischfarbe mit hellerem Nahtwulst und hellerem Schnabel.

Alle Stücke stammen von Gr. Popo. 
Nr. 14591. Dezbr. 1887. Salzwasser, 8 Faden, Schlickgrund. I Stück, in Spiritus.

Figg. 8, 8 a. $11-29,6-16,9-9,4$.

Nr. 25481. Septbr. 1888. Salzwasser, 6 Faden. 3 Stücke.

10. $23,1-14,2-7,2$.

Nr. 25515. Septbr. 1888. Desgleichen. 1 'Stiick, mit Pagurus besetzt.

Fig. 8 b. $10^{1 / 2} \cdot 23,5-14,1-8,0$.

Nr. 26027. Septbr. 15. 1888. Salzwasser, 6 Fadell. 1 Stïck.

Ich widme diese Art meinem lieben Freunde PROF. DR. GEORG Pfeffer, Kustos an unserm Museum.

\section{Tomella leschliei mihi.}

Figg. 9, 9 a, b.

Gehäuse spindelförmig; ziemlich glänzend, Gewinde mit etwas eingebogenem Seitenkontur. Schnabel mäßjig deutlich von der Wölbung der letzten Windung abgesetzt. Die $9-9 \frac{1}{2}$ Windungen sind anfangs durch einen Nahtwulst getrennt, der aber schon bei der 2. Windung gekielt ist, dam rasch flach wird, so daf er sich schlieblich fast nur durch die reine weilje Farbe abheht. Es kommen aber anch Stïcke vor, bei denen. wie Fig. 9l zeigt, der Nahtwulst auf den letzten beiden Windungen wieder gekielt ist. Nach den $1^{11}$. g glatten Embryonalwindungen tritt die Skulptur auf. die aus den üblichen Falten besteht, welche sich zuerst iiber die ganze Tindung erstrecken. wemn sie auch, wie üblich, nach dem unteren 'T'eile zu am stärksten ausgeprägt sind, dann oben obsolet werden und bei der 4. oder 5. Windung ganz anthören. Die Spiralskulptur ist stark ansgeprägt, wird aber allmählich schwächer, so daß sie auf der letzten Windung nur noch stellenweise schwach zu erkennen ist. Der Schnabel ist wieder mit deutlichen. flachen. schmalen Reifen besetzt. Der oben an der Mündung anstretende Callus ist verhältnismäßjig kräftig. Mündung, Spindelpartie, Mundrand und Analausschnitt verhalten sich wie bei der Gruppen-Diagnose geschildert.

Die Färbung ist durchscheinend weiß, mit rein weißem Nahtwulst, die oberen Windungen erscheinen z. T'. graubläulich (? durchscheinende Leber).

Die Stiicke sind leider alle tot bei Accra gesammelt, z. T. waren sie mit Pagurus besetzt, doch zeigt der Glanz des Gehäuses, daß die 
Färbung nicht durch Terwitterung beeinflußt ist. Es ist wahrscheinlich, daß keines der Stücke ausgewachsen ist.

$$
9^{1} / 2-18,7-11,6-6,3 .
$$

Ich widme diese Art dem Verwalter unserer Conchylien-Sammlung, DR. MAX LESCHKE, dem ich durch stets bereite Auskunft, besonders über die einschlägige Litteratur, zu besonderem Dank verpflichtet bin.

\section{Gruppe Perrone Schum.}

C. F. Schumacher gibt in seinem Essai d'un nouveau Système des hahitations des Vers testacés, Copenhagen 1817. pag. 218, folgende Diagnose der von ihm aufgestellten Gattung Perrona: Testa turita. Apertura ovalis; rostrum breve, subrecurvum; canalis apertus: labium externum tenue acutum postice excisum: labium internum obliteratum. Columella subflexuosa, oblique carinata, obscure umbilicata. Typus: Perrona tritonum = Murex perron CHejn., vol. X, pag. 278, Tab. 164, Fig. 1573-74. La figure est très mal faite, comme on n'y voit pas la forme naturelle de la columelle, ni celle du bec, aussi peu que son canal et l'echancrure de la lèvre externe; et même la déscription a des défauts considerables."

ScHUncachers Diagnose enthält nichts über die Skulptur, aber aus der Beschreibung der Form, besonders weil er den Schnabel kurz nennt, und aus der Kritik, die er an CHEגLNTTZens Figuren und Beschreibungen ïbt, scheint mir hervorzugehen, daß ihm eine von Murex perron CHEMN. abweichende Form, und zwar die spirata LA». vorgelegen hat. "Da nun sein Name der Art "Pervona tritonum" älter ist als der LAJARCKsche (1822), so muißte der letztere nach den aufgestellten Regeln eingezogen werden, wenn sich bestimmt nachweisen ließe, daß beide Arten identisch sind. Yarh ringe\%ogenen Erkmndigungen befindet sich der ScHudAcHeRsche Typus nicht im Kopenhagener Museum, von wo mir durch das freundliche Entgegenkommen von Dr. AD. JENSEN der CHEnINITsche Typus von Murex perron zur Kenntnisnahme zugeschickt wurde, auf den ich noch zurückkomme. Da nun SCHUMACHER seine Form weder abgebildet, noch genaner gesagt hat, worin demn der Unterschied der ihm vorliegenden Form mit der von ihm kritisierten CHEInITZschen besteht, da außerdem die LAALARCKishe Art schon dureh die Abbildungen in der Encyclopedie und in KIENER, die nach den LAMARTKichen (higinalen gemacht sind, festgelegt und iiberall als spirata eingebürgert ist, so darf man wohl den SCHUMACHERschen Namen ad acta legen, bleibt dem Autor doch die Ehre, den Gattungsnamen gegeben. zu haben. Nur bedarf die Diagnose der Gattung noch einigel Erö̈nzmgen, denn FIsthers kuze Diagnose 1. c. p. 5.90 bezieht 
sich nur auf die Unterschiede von Clavatula. Desgleichen die Erörterungen von Cossmann l. c. p. 68, der auch Perrona für eine Sektion von Clavatula hält, und der den SCHLHACHERschen Typusnamen tritonum dem LAMARCKschen voranstellt. 'TRYON gibt auch nur, ähnlich wie FISCHER, Unterschiede von Clacatula an, wobei er die Worte „or smooth" hinzufügt, weil el lineata zu Perrona zählt.

\section{Gruppe Perroma Schum. emend.}

Gehäuse spindelförmig bis getiirmt, festschalig, mattglänzend. Die Windungen setzen sich im Hauptteile mehr weniger deutlich treppenförmig voneinander ab. Sie sind anfangs durch einen Wulst voneinander getrennt, der sich später in eine Abdachung umbildet, die entweder nur durch eine etwas verdickte Kante in die wenig eingebogene IVindungswand übergeht, oder sich durch eine mehr weniger stark vorspringende Leiste von dieser abhebt. Die letzte Windung erscheint unten beim Übergang in den sich deutlich abhebenden Schnabel durch einen mehr weniger deutlichen Wulst mehr weniger kantig. Der Schnabel ist wie bei Tomella unten wulstig umgeschlagen. Mündung, Mundrand mit Einschnitt, Spindelpartie und Deckel verhalten sich ganz ähnlich wie bei Tomella.

Die ersten $1^{1 / 2}$ Windungen sind glatt, dann folgen neben einer mehr weniger deutlich ausgeprägten Spiralskulptur Falten in der Anwuchsrichtung, die entweder kaum hervortreten (Fig. 11a) oder nur in ihrem unteren Teile als schräge Falten an der Naht ausgebildet sind und sich nur auf ein paar Windungen erstrecken (Fig. 12), oder endlich diese schrägen Falten an der Naht erstrecken sich auf eine größere Anzahl der Windmogen und beeinflussen stellenweise sogar die Nahtabdachmo (Fis. 10b). Der schuabel ist mit mehr weniger dentlichen und meln weniger zahlreichen Spiralleistchen besetzt. Der an der Naht verlaufende Callusstreifen im Innern des Gehäuses tritt auch hier zuweilen auf, wenn auch schwächer, und mïndet nie in den mehr weniger kräftigen Wulst aus, den man bei Tomella findet.

\section{Perrona perron CHEMN.}

Figg. 10, 10a, b.

Wie ich schon weiter vorne erwähnte, war man in Kopenhagen so liebenswiirdig, mir das (HEdxITZsche Original zu dessen Fig.. 1573- it anzuvertrauen, so daß ich es genau prüfen und neu abbilden konnte. Man sieht daraus, daf die CHEmxirzschen Figuren, was die Form anbetrifft. durchaus nicht schlecht sind. 
Ich will nun zunächst den CHejrvitzschen Typus ex Museo Spengleriano beschreiben. Das Stiick ist besonders groß, aber leider nicht gut erhalten, trotzdem es noch eine hell fleischfarbige Grundfarbe erkemnen liaßt. Der Wirbel ist ansgebrochen, und die Windungen zeigen dentliche Spuren von Verwitterung; auch der Mundrand mit dem Einschnitt ist ausgebrochen. Die unteren 5-6 Windungen zeigen eine etwas platte, zunehmend stark vorspringende Nahtleiste, während die letzte Windung unten, oberhalb des Schnabels, keine eigentliche Kante, sondern nur eine schmale, weißliche Spiralleiste zeigt, die durch schräge, brämliche Striche gegliedert ist (Fig. 10). Die weiblichen Spiralreifen auf dem schnabel treten dentlich hervor und stehen ziemlich weitlüufig.

Die Falten an den oberen Windungen werden in ihrem oberen Teil rasch obsolet, so daß nur kurze schräge Falten oberhalb der Naht nachbleiben, die sich bis anf die fünfte der erhaltenen Windungen erstrecken, und die zuletzt sogar noch auf dem oberen Teil der Nahtleiste bemerkbar sind (Fig. 10b). Von einer Spiralskulptur ist nichts zu erkemnen, was vielleicht mit dem schlechten Erhaltungszustand zusammenhängt. Das Innere der Mrundmg zeigt die Außenfärbung, der Spindelbelag ist nach unten dentlich abgegrenzt, die spindelhasis zeigt den iihlichen wulstigen Umschlag. Die Maße sind bei 9 erhaltenen Windungen folgende:

$$
35,0-19,4-13,7 \text {. }
$$

Wie schon gesagt, ist die Zeichnung der Figg. 1573-74 in der Form ganz gut, nur hat der Zeichner die Anwuchsstreifen senkrecht anstatt geschweift gezeichnet, und die Farben sind etwas uibertrieben. Es ist erklärlich, daß LAJIARCK bei Beschreibung seiner spirata sich, wenn auch mit einem? versehen, auf die CHEMNITZschen Figuren bezog, denn schon die stark vorspringenden Nahtleisten berechtigen dazı. Er, wie die ihm folgenden Autoren haben aber einerseits die bedeutend höhere letzte Windung und die fehlenden dunklen Flecke auf den spiralleisten. hesonders aber die Skulptur der oberen Windungen, unberïcksichtigt gelassen, in der aber, wie aus meinen Abbildungen Figg. 10b und 11a ersichtlich ist, doch ein wesentliches Merkmal zur Unterscheidung der beiden Arten liegt.

Wenn einerseits es für mich keinem Zweifel unterliegt, daßs beide Formen zu tremnen sind, so muß es andererseits auffallen, daß, nachdem nummehr der CHEuñTzsche Typus genaner als bisher gekemnzeichnet ist, er als Unikum dazustehen scheint. Vielleicht gelingt es aber nummehr, gleiche Stücke auch in anderen Sammlungen aufzufinden, wo sie vielleicht, sei es unter der Bezeichnung spirata LAM., sei es unter perron oder perroni, unerkamnt liegen. Der von CHEגINITZ angeführte Fundort „Südmeer" dürfte ebenso falsch sein wie der von LAMARCK für seine spirata angeführte "Mer de la Chine". Es ist ja leider nicht mehr nachzuweisen, 
wo diese Typen gefunden sind; jedenfalls muß für perron ('HEys. angenommen werden, daß er von einem anderen Fundorte stammt als die hier folgenden Formen.

Es ist hier noch darauf hinzuweisen, daß WEINKAUFF 1. c. perron ('HEur. allerdings gesondert anfühnt, el fügt aber perronii REEvE und tritomm ScHUII. als Synonyme an, und gibt dazu als Abbildung die REEvEsche Fig. 94. Er fïgt hinzu (p. 119), daß er vorläufig nicht davon iiberzengt sei, daß perron und spirata verschiedene Arten seien. TRYON führt ebenfalls perron (HEMx. gesondert auf, aber auch unter Bezugnahme auf REEVEs Fig. It von perwnii. Er fügt damn die unerklïrliche Bemerkmg hinzu, daß er perron für eine Zwischenform von lineata und spirata halte.

\section{P. perron var. reevei mili.}

Figg. 13, 16, 17, 18 .

REEVE, Icon., Fig. 94, Pleurotoma perronii.

G. DUnker, 1S53. Index MIolluscorum über das von DR. TANs gesammelte Haterial. Pag. 27 wird $P l$ spirata subfossil bei Loanda gefunden aufgeführt, das den kurzen Bemerkungen nach vielleicht hierher gehört; vielleicht ist es auch eine spirata var. minor.

JLartini \& Chems., II. Edit., p. 123. Clavatula perron nach ReEves perronii, Fig. 94. TRYon 1. c. p. 232, Taf. S, Fig. S, ebenfalls als Perrona perron CHeirs. nach REEves perronii, Fig. 94.

F. P. MLARRAT, Quarterly Journal of Conchology, Vol. I, p. 240, führt perronii Chenn. (?) als unter dem Material befindlich auf, das KAPT. DAVIS zwischen Madeira und dem Golf von Guinea gesammelt hat. Es muß fraglich bleiben, ob damit perronii REEvE oder perron CHENs. gemeint ist.

Wir besitzen von dieser Form ein reiches, von Kapt. HuPFER gesammeltes Material, das, wenn auch in der Form teilweise zu der REEvEschen Fig. 94 passend, doch in Färbung und Skulptur abweichend erschien, so daß ich der Sicherheit halber zwei etwas verschiedene Stiicke an Herm EDGAR A. SMITH nach London sandte, wo sich das REEvEsche Original befindet. Er schrieb mir darauf das Folgende: "Your two shells certainly belong to this species (perronii Reeve). Reeve does not refer to the fine intercalations at the suture nor at the keel at the middle of the body-whorl although they are present in his type, yet you will notice that they are indicated in his figure 94. - Reeves type of perronii is a faded shell and only shows traces of the reddish markings upon the Keels which are more evident in your specimens."

Nachdem dadurch die Identität unseres Materials mit REEves perronii ertriesen ist, kann ich num im Angesicht des CHEIrNITzschen Typus ron perron feststellen, daf es sich bei der erstgenannten Art um eine abweichende Form liandelt, die ich vorläufig nur als Tarietät absondere, und 
zwar mit der Bezeichnung reevei, weil der Name perronii von REEvE nach perron falsch gebildet wurde, und an sich schon, mehr aber noch in seiner Terbesserung in perron, nicht bleihen kann, sobald nachgewiesen ist. dafs es sich dabei um eine abweichende Form handelt. Ua der Fundort der perron CHEMr. unbekannt ist, so läbt sich nicht entscheiden, ob die var. reevei eine sog. Standorts- oder geographische Varietät ist. Um geringere Abweichungen, wie sie bei jeder Art innerhalb verwandter Lokalitäten vorkommen können, handelt es sich hier jedenfalls nicht.

Die vorliegende Form liegt in gut erhaltenen Stücken vor, die, wenn sie auch natioliche Verschiedenheiten in Form- und Sknlptur-Nuancen anfweisen, doch in den Hauptcharakteren absolute Übereinstimmung zeigen.

Die Abweichungen von perron bestehen in folgendem: Das Gehäuse ist kleiner, die Windungen setzen stufenförmig voneinander ab, aber die Abdachung von der Naht ist steiler als bei perron und endet nur in eine schwach wulstige, nicht lamellenartig vorspringende Kante. Die Skulptur ist dieselbe, nur schïrfer ausgerrägt, was vielleicht durch den schlechten Erhaltumgsustand des Typus ron peron erklärt sein dürte. Auch hier treten die nur in ihrem unteren Teil anf den mittleren Windungen erhaltenen schrägen Falten oberhalb der Naht individuell auch etwas auf die Abdachung ibber, sie bleiben ferner auch individuell noch bis auf die letzte Windung erhalten, während sie im allgemeinen frïher aufhören und sich nur dadurch bemerkbar machen, daß am unteren Teil der letzten Windung die durch eine schmale weißliche Leiste gebildete Kante stellenweise durch Einkerbungen gegliedert erscheint. Ich gebe nachstehend eine genamere Beschreibung der Skulptur. Auf die ersten 1\% etwas bräunlichen, glatten und glänzenden Embryonalwindungen (vergl. Fig. 17) folgen auf etwa 2 Windungen von Naht zu Naht reichende, aber immerhin nach unten zu verstärkte Falten, die in der Anwuchsrichtung geschweift sind. Damn werden die Falten oben obsolet, und es bleiben nur die unteren Reste derselben oberhalb der Naht ibrig, die dann von der 7. Windung an ganz verschwinden, weil sie unter die Naht geraten. Wie gesagt, individuell bleibt noch mehr von ihmen erhalten, so daß sie dann noch bis zuletzt oberhalb der Naht sichtbar bleiben, dann aber auch auf der letzten Windung noch an stelle der meren Kantung erhalten b] (uben (Figg. 1\%. 18). Ton Spiralskulptm bemerkt man nur individuell auf den oberen Windungen unterhall, des Valitwulstes Furchen, damn auch wieder oberhalb des Schnabels (Fig. 13).

Die Färbung ist hellbräunlich-rosa, die Kanten sind weiß mit Fleiken oder Striemen der Grundfarbe. Die teilweise durch die Anwuchsstreifen in Körner anfgelösten Reifen auf dem schnabel sind weif und der untere Wulst des Schnabels ist ebenfalls weißlich. Die Zahl der Reifen auf dem Schnabel besteht aus 5 bis 7 stärkeren und dazwischen noch mehreren 
feineren. Das Innere ist mit einer dünnen weißlichen Schmelzschicht belegt, durch welche die äußere Färbung durchscheint. Der Spindelbelag ist dünne, nur nach unten etwas dicker und rosa gefärbt. Die ïbrigen Charaktere entsprechen der Gruppenbeschreibung.

\section{Fundstellen:}

1. Black Point. Nr. 26874. 16. 4. 1889. Salzwasser, 5 Faden. 7 Stuicke, mit Pagurus besetzt.

$$
\text { 8. } 22,5-14,8-9,3 \text {. }
$$

2. Setta Cama (Setta Kama). Nr. 21 254. Mai 23. 1891. Salzwasser, 8 Faden, Schlammgrund. 3 Stücke, davon 2, die anf dem unteren Teil der letzten Windung noch die schrägen Falten zeigen, von denen 1 Stïck nach London zum Vergleich mit perronii REEVE gesandt wurde (Figg. 13, 18).

$$
\text { Fig. 13, 18. Erhalten } 8 \frac{1}{2}-24,2-14,5-9,9 \text {. }
$$

3. Fluf Nyango.

$$
\text { " } 7-26,9-16,1-10,3 .
$$

Nr. 26774. 1888. Salzwasser, 6 Faden, weicher Grund. 2 jüngere Stiicke.

Nr. 26919. April 1889. Salzwasser, 6 Faden, Mudd-Grund. 4 Stücke, ziemlich schmal, wie Fig. 18, aber unten olme Falten, sondern mit einer in Flecke abgeteilten weißen Leiste, wie Fig. 16.

4. Loango.

$$
8^{1 / 2}-23,5-13,7-8,7 \text {. }
$$

Nr. 24399. Juni 4. 1888. Salzwasser, 5 Faden, Sand und MuschelGrund. 1 Stiick, unten wie Fig. 16.

Nr. 21255. Mai 1891. 13 Faden, Schlamm-Grund. 6 Stücke (3 mit Pagurus besetzt), wie die vorangehenden.

Nr. 20551. April 16. 1889. Salzwasser, $4^{1 / 2}$ Faden. 1 Stiick, wie die vorangehenden.

Nr. 24412. Juni 4. 1888. Salzwasser, 5 Faden, Sand- und MuschelGrund. 3 Stuicke, mit Pagurus besetzt.

$$
\text { Fig. 16. } 9-29,0-17,3-11,5 \text {. }
$$

5. Landana.

$$
\text { "17. } 6 \frac{1}{2}-11,4-7,6-5.0 \text {. }
$$

Nr. 26942. April 18. 1889. Salzwasser, 4 Faden, seln weicher Schlamm. 2 Stïcke, davon 1 jung, wie Figg. 16, 17.

Nr. 26936. April 16. 1889. Salzwasser, 4 Faden, sehr weicher Schlamm. 1 Stiick, mit Pagurus besetzt, wie Fig. 16. 
6. Cabinda (Kabinda).

Nr. 26766. 1888. Salzwasser, $3^{3} / 4$ Faden. 1 Stiick, schlank, wie Fig. 18, aber unten keine Falten, sondern eine gegliederte weiße Leiste.

$$
9-27,4-16,6-10,1 \text {. }
$$

Nr. 26 777. 1888. Salzwasser, $3 \frac{3}{4}$ Faden. 2 jüngere Stïcke, unten mit schrägen Falten.

Nr. 26600 . Febr. 15. 1889. Salzwasser, $3^{3 / 4}$ Faden. 3 Stiicke, mit Pagurus besetzt.

7. Kinsembo (Quissembo). Nr. 24482. Juni 4. 1888. Salzwasser, 7 Faden, Mudd-Grund. I Stück, wie Fig. 16.

8. Ambris (Ambriz). Ohne Nummer. Novbr. 1890. Salzwasser. 7 Faden. 3 Stücke, typisch, wie Fig. 16.

$$
\text { Erhalten } 8-24,4-15,3-11,0 \text {. }
$$

9. Ton Hupfer. Nr.21254. Ohne weitere Bezeichnung als WestAfrika. 1 schlankes Stiick.

$$
\text { Erhalten } 6^{1 / 2}-22,: 3-13,5-9,3 \text {. }
$$

Dies Stïck wurde zusammen mit dem unter 2. verzeichneten Stiick nach London geschickt, von dem es sich nur dadurch von Fig. 18 unterscheidet, daß es kleiner ist und unten keine Falten, sondern eine in Flecke aufgelöste weiße Leiste hat. Es ist der Fig. 94 von REEvEs perroni am ähnlichsten.

Man sieht aus den vorstehend verzeichneten Fundorten, daß diese Form in den westlicheren bezw. nördlicheren Bezirken nicht rorzukommen scheint.

\section{Perrona spirata LAII.}

LAMARCK 1. c. vol. VII, p. 93, mit fraglicher Bezugnahme auf MART. \& ChEMN. l. c. Figg. 1573-4.

Derselbe, Edit. Deshayes 1. c, vol. IX, p. 348, mit besonderem Hinweis auf Encyclop.

Taf. 440, Figg. 5 a, b. Von den weiteren Hinweisen führe ich nur die mit Abbildung versehenen an.

DAviLA, vol. I, Taf. 5, Fig. L, entspricht wohl der kleineren Form.

Wood, Index, Taf. 27, Fig. 120, dürte eine Kopie der Chemnitzschen Figuren von perron sein, also nicht hierher gehörig.

KieNER, Icon., p. 46, pl. 5, Fig. 2.

REEVE, Icon., Fig. 44, scheint eher meine var, minor. 
IVEINKAUfF bildet in ILART. \& Chemn., II. Edit., auf Taf. 26 in Figg. 2, 3 ein Stück $a b$, das wohl eine Lokalvarietät sein dürfte. Ich komme darauf noch zurück. Tryon 1. c. p. 232, Taf. 8, Fig. 5.

Es ist mir leider nicht gelungen, die LAMARCKschen Originalstïcke, welche sich im Genfer Museum befinden, zur Ansicht zu erhalten, doch hatte der Erste Assistent. Herr Dr. E. F. WEBEr, die Freundlichkeit, mir iiber einzelne Punkte erbetene Auskunft zu geben.

1. Ich hatte meine Zeichnungen der oberen Windungen von perron CHEMn. und spirata LAм. bezw. die hier wiedergegebenen Figuren $10 \mathrm{~b}$ und 11 a eingeschickt. Danach sollen die von spirate (nach meiner var. minor) zu den LAJARCKschen Stïcken passen, und nicht die von perron. Man schickt mir eine sechsfache photographische Tergrößerung der oberen Windungen von dem Stücke, welche der KTENERschen Tarietät, Fig. ${ }^{1}$, entspricht, die aber maßgebend für die anderen Stücke sein soll. Danach sind allerdings nur stellenweise Spiralfurchen, und nur Anwuchsstreifen, keine Falten, zu erkennen.

2. Meine fragliche Annahme, ob nicht etwa diese KIENERsche Tarietät, Fig. $2^{1}$, die einen längeren Schnabel als das Stück Fig. 2 zeigt, zu perron CHEMN. passen kömne, wird damit widerlegt, daß das Stück sich in nichts von den andern beiden Stïcken unterscheide.

3. KIENERS Abbildungen zweier der 3 in der LAMARCKschen Sammlung vorhandenen Stücke seien exakt, nur sei die Färbung übertrieben. Das dritte Stïck entspreche der REEvEschen Fig. 44 zu spirata. Es scheint dies Stïck also zu meiner var. minor zu gehören, wenn es nicht etwa jung ist. Die Abbildung der spivata in MART. \& CHENN., II. Edit., Taf. 26, Figg. 2, 3, wird als viel schlechter als die von KIENER genannt, was sich wohl daraus erklärt, daf dies Stück allerdings einer abweichenden Form entspricht, die ich mit var. weinkauffi bezeichne. Für die von KIENER beschriebene und abgebildete spirata werden 9-10 Windungen bei einer Größe von 16" $=36,1 \mathrm{~mm}$ angegeben.

Aus der gegebenen Auskunft erhellt, daß 1. die KIENERschen Abbildungen für den LAґARCKschen Typus maßgebend bleiben. 2. Daß perron CHEMN. von spirata LA\. verschieden ist. 3. Daß der längere schmabel der KiExERschen Fig. ') nicht als [nterscheidmgsmerkmal von perron rHEys. dienen kann, wenn er nicht etwa rom Zeichner übertrieben ist.

WVie schon frïher bemerkt, kann die LAJARCKsche Angabe des Fundortes "Mer de la Chine" wohl kaum richtig sein. Es bleibt also der Zukunft überlassen. ähnlich grofje Stïcke anfanfinden und den richtigen Fundont darnach festzustellen. Die Art steht nach dem mir vorliegenden Material ebenso isoliert da, wie der CHesnnTzsche Typus von perron, so daß es wohl berechtigt ist, vorläufig die nachfolgende Form als Varietät abzulsondern. 


\section{P. spirata var. minor mihi.}

Figg. 11, 11a, 12, 14 .

Diese kleinere Form liegt nur in wenigen Stücken vor, die in ihrer Färbung voneinander abweichen. Die oberen Windungen, wie sie in Figg. 11 a und 12 wiedergegeben sind, zeigen vorwiegend eine spiralskulptur. die sich später verliert, daneben in den auf die $1 \frac{1}{2}$ glatten Embryonalwindungen folgenden 2 Windungen stïrkere Anwuchsstreifen oder auch deutliche kurze Falten oberhalb der Naht. Die Leiste an der Naht ist mehr weniger stark rorspringend. Die untere Kante der letzten Windung ist wulstig. Mündungspartie und Spindelpartie entsprechen den Angaben für die Gruppe. Die Spiralleisten am Schnabel treten nicht so hervor und sind nicht so zahlreich wie bei perron CHEsn. var. reevei, von der sich diese Form schon durch die vorspringenden Nahtleisten wie durch die Färbung und durch die Skulptur der oberen Windungen leicht miterscheidet.

Soweit es das Hupfersche Material zeigt, kommt die Form zusammen mit $P$. perron $v$. reevei vor.

1. Setta Camma (Setta Kamma). Ohne Nummer und nähere Bezeichnung. 1 Stïck wie das nachfolgende, mit abgebrochenem Schnabel.

2. Ambris (Ambriz). Novbr. 1890. Salzwasser, 7 Faden. 2 frische Stiicke, bräunlichgrau, nach oben mehr grau, nach dem Schnabel weißlicher werdend. Die Zone an der Naht auf und unterhalb der Spiralleiste sowie an der unteren Kante der letzten Windung ist weif, mit weitläufigen, kastanienbraumen Flecken verziert. Auf der letzten Windung treten zwischen den weißen Zonen anf dem grauen Grunde einige brame Striemen in der Anwuchsrichtung auf.

Fig. 11, 11a. $10-26,7-14,3-10,7$.

3. Aus dem Berliner Museum von DUxKER stammend, auf der Etikette ist Gambia bemerkt. Das stück ist nicht gut erhalten, entspricht aber im allgemeinen den vorausgehenden, nur zeigen sich oben in den 2. und 3. Windungen Reste von kurzen Falten oberhalb der Naht (Fig. 12). Es sind nur ein paar schwache Spiralleisten am Schnabel erkennbar.

$$
\text { Fig. 12. } 10-22,2-11,2-9,2 \text {. }
$$

4. Aus der LöBBECKEschen Sammlung zur Ansicht erhalten:

a) 1 Stück LIschKE leg., Senegal-Küste, wie das Stück des Berliner Museums mit Falten auf der 2. und 3. Windung.

$$
9^{1 / 4}-16,6-8,7-7,1 \text {. }
$$

b) 2 Stiicke von v. LENNEP stammend, Afrika, entsprechen auch dem vorangehenden. Das eine zeigt Falten auf der 2. und 3. Windung, das andere keine. 


$$
\begin{aligned}
& 10^{1 / 2}-2 \cdot 2,9-11,3-9,2 . \\
& 10-22,3-11,6-9,2 .
\end{aligned}
$$

5. ex Koll. ScholviEN. 1 Stiick von West-Afrika, durchaus den sub 2 verzeichneten entsprechend; es zeigt oben Spuren von kuren Falten oberhalb der Naht wie Fig. 12.

$$
\text { Erhalten } 9-24,2-13,1-11,1 \text {. }
$$

Ein zweites Stïck ist ganz elfenbeinweif, schlecht erhalten, Mundrand etwas ausgebrochen, Wirbel fehlt. Das Stïck ist nur deshalb interessant, weil es ein verhältnismäßig hohes Gewinde hat und schlank gebaut erscheint. Auf dem Schmabel sind deutlich 10-11 Spiralleisten sichthar. oben lassen sich keine Falten erkennen.

$$
\text { Erhalten } 10-26,0-13,7-10,3 \text {. }
$$

6. ex Koll. FщuY. Nr. 18743. 1 Stiick. West-Afrika. Das Stück ist nicht gut erhalten, trotz des eingeklebten Deckels. Es erscheint grau. mit weißlichen Spiralleisten, die stark vorspringen. Von den braunen Flecken und Striemen ist nichts zu erkennen, von Spiralleisten am Schnabel nur eine Spur; Falten auf der 2. und 3. Windung sind nicht vorhanden.

$$
\text { Fig. 14. } \quad 9^{1 / 2}-27,3-14,8-12,0 \text {. }
$$

7. Auf eine Anfrage bei Herrn EDGAR A. Sumt bekomme ich die Auskunft, daß die im Londoner Musem befindlichen Stïcke von spirnta ebenfalls schräge Falten auf der 2. und 3. Windung oberhalb der Naht zeigen.

\section{P. spirata var. weinlearfiti mihi.}

Figg. 15, 15 a.

Dies von WEINKAUfF in MART. \& CHEגז., II. Edit., auf 'T'af. 26, Figg. 2, 3, für $P$. spirate nicht besonders gut abgebildete Stuick ist aus der LöBBECKEschen Sammlung und stammt ron S'HEEPIAKER, wie die Etikette besagt; Fundort Senegalküste. Es entspricht in der Größe dem LAMARCKschen 'Typus, hat aber eine entschieden getiirmte Form mit kiuzerer letzter Windumg. Das stiick ist schlecht erhalten und mit einem (?) Firnis überzogen. Der Wirbel ist ausgebrochen. Die erhaltenen oberen 5) Windungen zeigen oben und unten einen Wulst (Fig. 15a), letzterer könnte auf kurze Falten oberhalb der Naht verweisen, ist vielleicht aber auch typisch. Damn tritt die immer deutlicher ausgenügte Abdachung mit vorspringender Leiste ein. Die letzte Windung ist unten nicht kantig, nur tritt die Wölbung sehr plötzlich in den schnabel ïber. der mit ein yaar schwachen Spiralleisten besetzt und unten wie iiblich wulstio 
mungedreht ist. Sjuralfurchen sind auf den mittleren Irindungen unterhall) der Nahtleiste erkemnbar. Die Färbung ist oliv-bräunlich, die Nahtleisten etwas heller, auf denen sich noch dunklere Flecke erkemnen lassen, die anf der letzten Windung in bräunliche Striemen in der Anwuchsrichtung auslanfen.

Figg. 15, 15a. Erhalten $11-38,0-19,0-15,0$.

Auch hier haben wir es vorläufig mit einem Unikum zu tum, von dem wenigstens der Fundort angegeben ist.

Ich bin mir wohl bewußt, daß die in der Gruppe Perrona vorgenommene 'T'rennumg der' verschiedenen Formen keine endgültige sein wird. Es wäre sehr wohl möglich, daß man der mit dem ältesten Namen perom CHEmx. bezeichneten Form alle übrigen hier anfgezählten Formen muterzuordnen hätte, besonders wemn der Unterschied in der Skulptur der postembryonalen Windungen durch erweisbare Übergänge an weiterem Material sich als hinfällig erweisen sollte. Dann würde die eine Formenreihe von perron aus nach spirata und ihren Tarietäten minor und veinkauffi gehen, die sich durch die vorspringende Leiste an der Naht auszeichnet, während eine andere Reihe nach perron var. reevei (perronii REEVE) geht, bei der diese vorspringende Leiste fehlt.

Vorläufig schien es mix nach dem vorliegenden Material g'eraten, die 'Tremmung der verschiedenen Formen so wie geschehen vorzunehmen.

TRYox fülnrt unter Perrona außer den hier eingehend behandelten Arten noch taxus. CHEIN. vom Kap der guten Hoffnung, obesa REEvE von West-Afrika und monite TAL. von Australien auf. Die beiden erstgenannten Arten möchte ich weder zu Perrona noch zu Tomella zählen, sie bilden zusammen mit der später beschriebenen Perrona subspirata von MLRTExs aus der großen Fischbai (Dentsche Tiefsee-Expedition der Taldivia, p. 6) eine Gruppe von Clavatula-Arten, auf die ich hier nicht näher eingehen kamn, da mir gut erhaltenes Material fehlt, um obere Windungen und Skulptur beurteilen zu können, der ganze Habitus weicht aber entschieden von dem der Perrona- und Tomella-Arten ab.

Die Clavatula monile YAL. kamn schon des Fundorts halber nicht gut hierher gehören, was auch durch die Abbildung unterstiitzt wird. Man hat vielfach monile TAL. und Quoyi DESM. für identisch gehalten, TRYON hält sie für verschieden und fühnt Quoyi, wie sie von REEvE und TVEINKAUFF gedentet wird, unter den Surcula-Arten mit kurzem Kanal 
auf. Diese Quoyi gehört aber ebenfalls nicht zu Perrona oder Tomella. Ich mus hier noch hinzufïgen, daß trotzdem sich unter dem Hurfersehen Material viele der größeren Surcula- und Pusionella-Arten befinden, die obesa REEVE, die doch auch von West-Afrika stammen soll, fehlt, die also in den von HuPFER besuchten Stationen und in den von ihm verzeichneten Tiefen nicht vorkommen dürfte. Das Gleiche gilt von den z. Z. als Unica zu betrachtenden Formen $P$. perron CHEMn., $P$. spirata LAM. typica und ihrer var. weinkauffi.

Hiermit kann ich diese Studie schließen, die dank dem ihr zugrunde liegenden reichen und sorgfältig gesammelten Material in mancher Beziehung sowohl über die Abgrenzung der Arten wie auch ihre natürliche Gruppierung zu anderen Ergebnissen führen mußte, als es bisher auf mangelhafter Grundlage möglich war. Wenn es mir bei dieser Gelegenheit gestattet war. für einioe bisher mißdentete oder doch fragliche typische Stücke in Wort und Bild mafgebende Auskmft zu geben, so rerdanke ich das dem freundlichen Entgegenkommen der Herren JExsEx in Kopenhagen. SuIth in London. Teber in Genf. THIELE in Berlin und Wexi in Diisseldorf.

Mitte April 1912. 


\section{Inhaltsverzeichnis.}

Die mit * bezeichmeten Arten sind Synonyme.

\begin{tabular}{|c|c|c|c|c|}
\hline & & Seite & & Seite \\
\hline Gruppe & Perrona Schum... & 12 & Perrona taxus Chemn.... & 22 \\
\hline Perrona & a monile Val. ..... & 22 &,$\quad *$ tritonum Schum....... & 12 \\
\hline$"$ & obesa Reeve....... & 22 & & \\
\hline , & perron Chemn.......... & 13 & Gruppe Tomella Swainson. & 3 \\
\hline$"$ & perron v. reevei Strebel & 15 & Tomella hupferi Strebel ........ & 9 \\
\hline 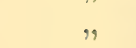 & *perronii Reeve......... & 15 & $" \quad$ " $\quad$ v. fusca Streb. . & 10 \\
\hline$"$ & spirata Lam. ........... & $1 \mathrm{~S}$ & leschkei Strebel......... & 11 \\
\hline " & $\eta \quad$ v. minor Strebel. & 20 & lineata Lam. .......... & 5 \\
\hline & $" \quad$ "weinkauffi & 21 & " $\quad$ v. castanea Lam. & 5 \\
\hline & subspirata v. Martens... & 22 & pfefferi Strebel.......... & 10 \\
\hline
\end{tabular}

\section{Erklärung der Abbildungen auf der Tafel.}

Figur

1, $1 \mathrm{a}, 1 \mathrm{~b}$

2

3

4, $4 \mathrm{a}$

5

$6,6 a, 6 b$

7. 7а

S, Sa, sb

9, $9 a, 9 b$

$10,10 \mathrm{a}, 10 \mathrm{~b}$

$11,11 \mathrm{a}$

12

13

14

$15,15 a$

16,17

18

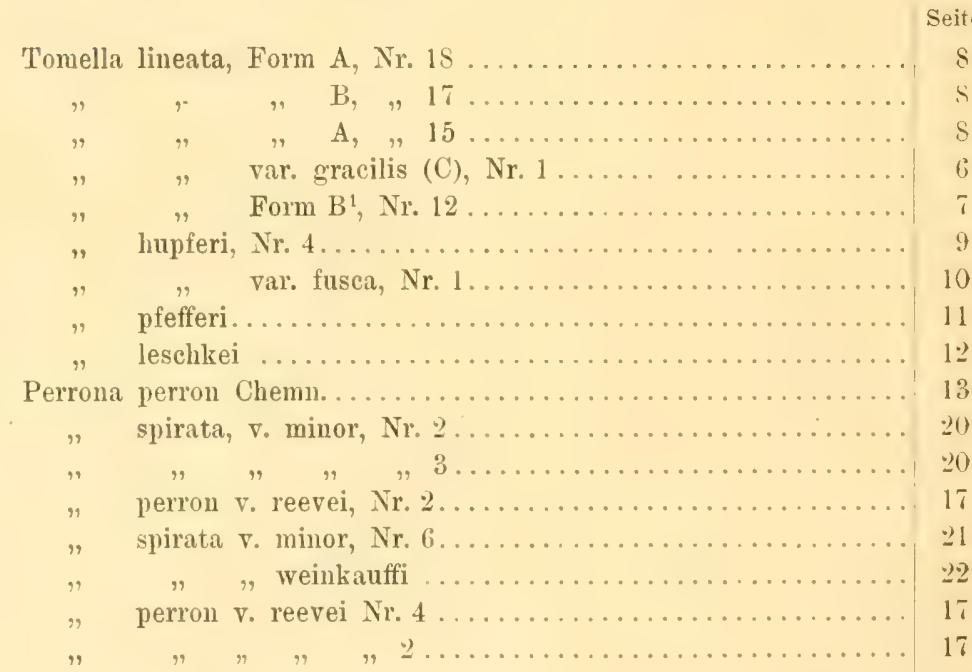

Eingegangen den 18, April 1912. 


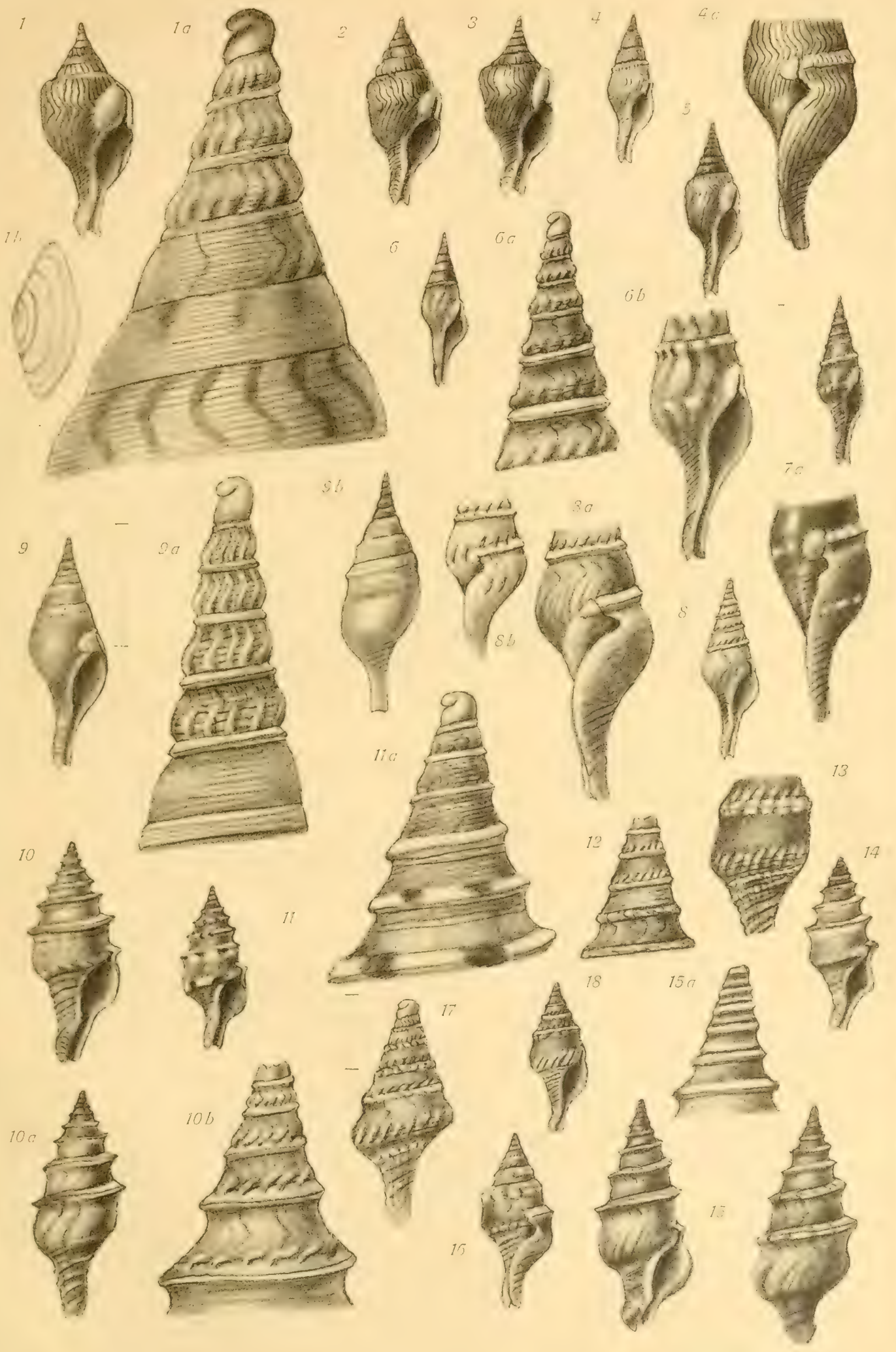





\section{Bemerkungen zu den}

\section{Clavatula-Gruppen Perrona und Tomella.}

Von

Hermann Strebel.

Mit e in er Tafel.

Aus „Mitteilungen aus dem Naturhistorischen Museum“. XXIX.

(2. Beiheft zum Jahrbuch der Hamburgischen Wissenschaftlichen Anstalten. XXIX.)

Hamburg 1912.

Kommissionsverlag von Lucas Güre \& Sillem. 






















